\title{
Norois
}

Environnement, aménagement, société

$211 \mid 2009 / 2$

Eau, pêche, tourisme rural, conflits d'usage

\section{La répartition des populations piscicoles en Creuse (Limousin), l'apport géographique des pêches électriques en tête de bassin}

Distribution of fish populations in the western part of the French Central Massif. The geographical contribution of electrofishing in headwater catchments

Laurent Touchart et Yann Clave

\section{OpenEdition}

\section{Journals}

Édition électronique

URL : http://journals.openedition.org/norois/2857

DOI : 10.4000/norois. 2857

ISBN : 978-2-7535-1559-8

ISSN : $1760-8546$

Éditeur

Presses universitaires de Rennes

Édition imprimée

Date de publication : 1 octobre 2009

Pagination : 23-36

ISBN : 978-2-7535-0937-5

ISSN : 0029-182X

Référence électronique

Laurent Touchart et Yann Clave, « La répartition des populations piscicoles en Creuse (Limousin), l'apport géographique des pêches électriques en tête de bassin », Norois [En ligne], 211 | 2009/2, mis en ligne le 01 octobre 2011, consulté le 01 mai 2019. URL : http://journals.openedition.org/ norois/2857 ; DOl : 10.4000/norois.2857 


\title{
La répartition des populations piscicoles en Creuse (Limousin), L'APPORT GÉOGRAPHIQUE DES PÊCHES ÉLECTRIQUES EN TÊTE DE BASSIN
}

\author{
LAUREnT TOUCharT \\ (Université d'Orléans) \\ EA 1210 CEDETE \\ 10 rue de Tours BP 46527 - 45065 OrLÉANs cedex 2, France \\ laurent.touchart@univ-orleans.fr \\ Yann Clave \\ Parc Naturel Régional de Millevaches en Limousin \\ Le Bourg - 23340 Gentioux-Pigerolles
}

\section{RÉSUMÉ}

Le département de la Creuse a une situation hydrologique de tête de bassin, que le bon état écologique des cours d'eau permet de considérer comme un espace de référence. Les pêches électriques sont utilisées de manière géographique pour cartographier la répartition des poissons représentatifs d'une certaine qualité des eaux et leur suivi pendant sept ans sur un vaste espace de $5600 \mathrm{~km}^{2}$. D'année en année, les espèces peu exigeantes (chevesne et vandoise) progressent de l'aval vers l'amont, tandis que les espèces les plus exigeantes (vairon) régressent pour ne plus subsister qu'en tête de bassin. Ces espèces sont les traceurs d'une dégradation des conditions physico-chimiques ou sédimentaires remontant d'aval en amont.

MotS CLÉ : tête de bassin - géographie piscicole - hydrologie - pêche - Limousin - Creuse

\section{ABSTRACT}

Distribution of fish populations in the western part of the French Central Massif. The geographical contribution of electrofishing in headwater catchments

The department of the Creuse is located in the highlands of the French Central Massif, where the headwater catchments constitute a reference area for the ecologically healthy small watercourses. Electrofishing is used here in a geographical way in order to chart the repartition of fish species representative of a certain water quality, covering a period of seven years and a large area of $5600 \mathrm{~km}^{2}$. Over the years, populations of the least sensitive species (chub and dace) progress towards upstream waters, while the most sensitive species (minnows) regress and only subsist in the headwaters. These species are indicators of a degradation of physicochemical or sedimentary conditions moving upstream.

KEY WORDS : headwaters - piscicultural geography - hydrology-fishing - French Central Massif 
La distribution spatiale des populations piscicoles est l'une des caractéristiques des cours d'eau les plus complexes à appréhender, dans la mesure où sa détermination se fait en général avec des méthodes ponctuelles, dont les données sont difficiles à replacer dans le continuum fluvial. Ce type de recherche participe à la définition de l'état piscicole des cours d'eau, dont le suivi permet de connaître les potentialités halieutiques et l'évolution de ces ressources. Dans le contexte de la Directive Cadre Européenne (DCE) de décembre 2000, pour une politique communautaire dans le domaine de l'eau, les enjeux sont devenus considérables, en particulier en termes de réalisation d'états des lieux dans chaque district hydrographique et de l'identification des pressions dues aux activités humaines. Or la dynamique spatiale des populations piscicoles est un indicateur d'autant plus pertinent de la qualité globale des cours d'eau qu'il se trouve en aval de la chaîne trophique. Pourtant, à part quelques éminents précurseurs (Bravard, 1987; Rougerie, 1993) et une exception (Berthold, 2003), les géographes français ont peu abordé la thématique du poisson indicateur, bien que certains travaux pluridisciplinaires, intégrant des géographes, aient frayé ce chemin (Amoros \& Petts, 1993, Wasson et al., 1993). De fait, la plupart des initiatives à ce sujet ont été laissées aux biologistes ou aux organismes techniques (Persat et Keith, 1997; Girard, 1998; Oberdorff et al., 2002a; Brun et Pinet, 2004), y compris en terme de cartographie (Keith et Allardi, 2001). Quant aux références géographiques concernant la pêche dans les eaux intérieures, elles sont rares, tant à l'étranger (Carré, 1978) que dans les eaux douces françaises (Ardillier-Carras, 1997). C'est pourquoi le but principal de cet article est d'apporter une contribution géographique, privilégiant la recherche à petite échelle cartographique et l'interprétation spatiale dans un domaine habituellement dominé par la mise au point des indices et la modélisation. Pour y parvenir, nous avons décidé de focaliser l'étude sur l'apport spécifique des pêches électriques eu égard aux autres méthodes de détermination de l'état des populations piscicoles.

\section{Le département de la Creuse, en Limousin : un site d'étude privilégié}

La recherche, puisqu'elle porte sur le réseau hydrographique de l'ensemble du département limousin de la Creuse, offre, d'une part, l'originalité des grandes dimensions de l'aire d'étude, d'autre part, l'intérêt d'un espace peu peuplé en tête de plusieurs bassins.

À la différence d'études biologiques ou hydrochimiques très précises, qui prennent pour témoin un petit bassin-versant expérimental à très grande échelle cartographique, il a été ici décidé d'embrasser un vaste espace de $5600 \mathrm{~km}^{2}$, dont le chevelu hydrographique s'allonge sur $5500 \mathrm{~km}$. Notre recherche à petite échelle cartographique s'appuie sur une entité administrative qui permet de collecter des données sur plusieurs bassins d'alimentation et régions géographiques, essentiellement la Haute Marche à l'ouest et la Combraille à l'est, dont l'intérêt justifie le choix de l'aire d'étude (fig. 1).

Outre sa taille, à l'échelle d'un département, la Creuse multiplie les atouts particuliers, qui légitiment de la sélectionner pour une première approche géographique de la distribution des populations piscicoles. Son intérêt majeur réside dans le fait que sa situation au nord-ouest du Massif Central place cet ensemble de plateaux à la source de nombreuses rivières, qui divergent ensuite, vers l'aval, pour arroser différentes régions. Le substrat cristallin et imperméable de ce massif ancien, qui fonde l'appartenance du terrain d'étude à l'hydro-écorégion des « terres granitiques du Massif Central » de J.-G. Wasson et al. (1993), favorise une assez forte densité de drainage de $0,98 \mathrm{~km} / \mathrm{km}^{2}$. La ligne de partage des eaux entre le bassin de la Loire et celui de la Garonne passant en Combraille méridionale, entre Crocq et Courtine, le département de la Creuse possède une limite fluviale majeure. Le département possède en effet le cours supérieur de la Vienne et les sources du Cher, ainsi que la partie amont d'affluents du Sioulet, se jetant ensuite dans l'Allier. Grâce à cette divergence hydrographique, le département de la Creuse arrose le Périgord, le Haut Limousin, le Confolentais, le Poitou, le Berry et le Bourbonnais. C'est le bassin de la Vienne qui est le plus représenté, couvrant dans le département $4128 \mathrm{~km}^{2}$. Drainée directement par la Vienne pour $73 \mathrm{~km}^{2}$ ou par l'intermédiaire de la Maulde $\left(185 \mathrm{~km}^{2}\right)$, du Taurion $\left(835 \mathrm{~km}^{2}\right)$ 


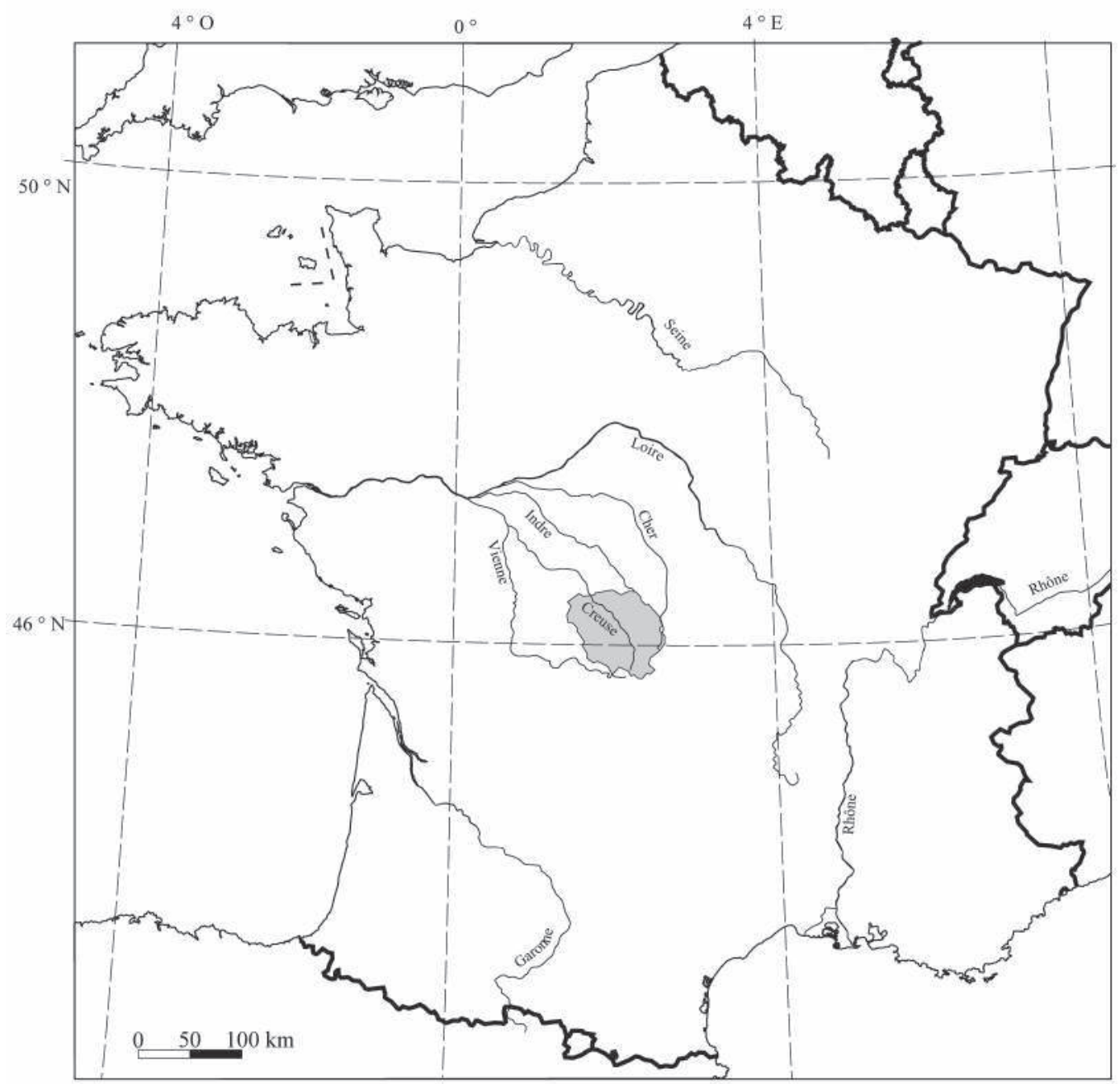

Figure 1 : Carte de localisation de l'espace étudié Studied area

et de la Creuse $\left(3035 \mathrm{~km}^{2}\right.$ ). Le secteur amont de la Vienne a été officiellement désigné comme bassin d'essai pour préparer le huitième programme d'intervention de l'Agence de l'Eau destiné à mieux définir la politique d'aide aux régions dites «têtes de bassin ». De plus, l'importance des ruisseaux et des têtes de bassin est désormais reconnue au niveau européen, par un programme LIFE (Fédération des PNR, 2007). Ainsi localisé à l'amont des bassins du Centre-Ouest de la France, le département a une responsabilité importante en terme de préservation de la qualité de ses eaux envers les régions situées en aval. En revanche, il n'est en aucun cas influencé par les eaux d'autres territoires et peut donc être considéré, à cet égard, comme un espace de référence, dans lequel les variables peuvent être isolées sans le bruit de fond ni les interrelations complexes inhérents aux espaces situés plus en aval.

La deuxième justification du choix de l'aire d'étude réside dans la très faible densité de population, puisque la valeur moyenne départementale de $22 \mathrm{hab} / \mathrm{km}^{2}$ est l'une des plus basses du territoire français. Extrêmement peu urbanisé et industrialisé, cet espace d'activités rurales exten- 
sives doit, a priori, présenter des eaux de bonne qualité, où la dégradation écologique est encore susceptible de se révéler, d'apparaître ou de se poursuivre à partir d'un état «originel » encore très reconnaissable. La Fédération de la Creuse (2008) a d'ailleurs identifié, dans son PDPG, « des contextes salmonicoles conformes » dans les parties amont des bassins de la Creuse et de la Mourne, et dans la partie aval de la Maulde, alors que l'essentiel du département se trouve en « contexte salmonicole perturbé ». En revanche, le « contexte dégradé » est pratiquement absent. Il est à noter que le suivi de l'état écologique des eaux répond au principe même de la DCE 2000, demandant la comparaison entre l'état des lieux de 2004 et celui de 2015. Dans cette confrontation à échéance de quelques années, la France aura très peu de territoires comparables à celui de la Creuse, où la répartition des poissons sensibles pourraient être proches d'une situation non perturbée.

Les aménagements hydrauliques perturbant l'état initial des cours d'eau ne sont cependant pas absents et il est utile d'en présenter la situation au milieu des années 1990, quand commence le dépouillement pluri-annuel de nos données. Les grands barrages concernent le cours principal de la Creuse, du Taurion, de la Maulde et, à la sortie du département, du Cher. Les étangs concentrent leur plus forte densité au nord-est du département et sont les moins nombreux au sud et à l'ouest (Bartout, 2006). Parmi les autres petits aménagements, les anciens moulins en forment la plupart. Ainsi, le SAGE du Cher amont (2007), qui déborde cependant largement en aval du département de la Creuse, a recensé ces derniers comme constituant près de la moitié des 237 ouvrages totaux de ce bassin.

Enfin, la troisième justification du site d'étude repose sur le fait que l'activité de pêche représente un poids d'une importance certaine dans cette région (Balabanian et Bouet, 1989). En 2000, les 37 Associations Agréées pour la Pêche et la Protection des Milieux Aquatiques (AAPPMA) creusoises regroupent 10400 pêcheurs dans un département qui ne compte pourtant que 120000 habitants. C'est que le nombre de cartes vendues est grossi par l'afflux touristique. En effet, $11 \%$ du total départemental correspondent à des cartes de pêche dites "vacances ", qui permettent aux estivants de pratiquer cette activité pendant deux semaines entre juin et septembre et $5 \%$ sont des cartes journalières autorisant les touristes à une initiation. L'attraction des pêcheurs extérieurs est indispensable, car la chute des effectifs locaux est forte et, pour les trois départements limousins, la baisse des adhérents a été de $35 \%$ en vingt ans (Ardillier-Carras, 1997). Sachant que la baisse a été de $50 \%$ depuis le milieu des années 1960 au niveau national (Brun et Pinot, 2004), la concurrence pour l'attractivité s'exacerbe. Dans l'optique du développement du tourisme rural en Creuse, la pêche est un produit d'appel essentiel, fondé sur l'importance des cours d'eau de première catégorie.

\section{La méthodologie des pêches électriques et les échelles géographiques}

À la suite, et parfois en parallèle, des études mettant en place l'ordination des cours d'eau sur des critères morphométriques (Horton, Strahler, etc.), les recherches concernant la zonation géographique des poissons en eaux courantes ont évolué, depuis la règle des pentes de Huet (1949), dans le sens d'une complexification. Les travaux de J. Illies $(1955,1961)$ et J. Illies et L. Botosaneanu (1963) restaient en grande partie fondés sur le débit et la vitesse du courant. Dès les années 1970, J. Verneaux (1977) classa les gradients des faunes benthiques et piscicoles d'amont en aval d'après la distance à la source, la température maximale de l'eau, la section mouillée et la dureté de l'eau. Puis le concept de continuum fluvial (Vannote et al., 1980) élargit les critères, associant, d'une part, la production primaire et les invertébrés, prenant, d'autre part, en compte les relations spatiales entre l'amont et l'aval, le cours d'eau et sa vallée, sa plaine alluviale et sa nappe. De ce point de vue, l'éclairement de la rivière et la pénétration de la lumière dans l'eau sont déterminants (Descamps et Naiman, 1989). Plus récemment, l'Indice Poisson se fonde sur la distance à la source, la superficie du bassin-versant, la largeur et la profondeur moyenne de la station, la température moyenne de l'air en janvier et juillet et l'appartenance à un bassin hydrographique pour modéliser les peuplements 
piscicoles (Oberdorf et al., 2002a, 2002b). D’une manière générale, la longue évolution des conceptions a été dans le sens de l’intégration de plus en plus de paramètres (Karr, 1981).

C'est grâce à ces avancées scientifiques longues de plusieurs décennies que certaines structures françaises liées à l'eau ont pu mettre au point des protocoles de suivi de qualité fondés, pour une certaine part, sur les emboîtements d'échelles géographiques. Pour les agences de l'eau, de même que pour le CSP (puis l'ONEMA), le niveau national, de plus en plus dépendant des directives de l'Union Européenne, est celui des enjeux politiques environnementaux les plus larges. Concernant le thème piscicole, il est celui du Réseau Hydrobiologique et Piscicole (RHP), du Suivi National de la Pêche aux Engins (SNPE) ou encore de la Station de Contrôle des Migrateurs (StaCoMi). Le contexte piscicole est un deuxième niveau géographique, qui correspond à l'aire de répartition fonctionnelle d'une espèce qui comprend l'ensemble des habitats nécessaires à l'épanouissement de la chaîne des fonctions vitales de la reproduction, de l'éclosion et de la croissance. Les Plans Départementaux de Protection du milieu aquatique et de Gestion des ressources piscicoles (PDPG), en lien avec le Réseau d'Observation des Milieux (ROM), mis en place par le CSP en 2002-2003, tentent de travailler, autant que possible, en utilisant la notion de contexte piscicole. Le PDPG de la Creuse définit ainsi 31 contextes (Fédération de la Creuse, 2008). Les deux espaces ne coïncident pourtant pas tout à fait, formant ainsi une distorsion géographique. Le troisième niveau utilisé pour l'administration et le suivi de la qualité piscicole des cours d'eau est le tronçon, qui constitue une unité linéaire homogène sur quelques kilomètres ou dizaines de kilomètres du point de vue de la morphologie fluviale. Le tronçon, ainsi utilisé par le Réseau d'Évaluation des Habitats (REH), sert de cadre à la description des liens entre l'état du chenal et des berges, la physico-chimie de l'eau et la composition des espèces. Le quatrième et dernier niveau est celui de la station. Par rapport aux autres, on peut le considérer comme ponctuel, bien qu'il soit en fait défini comme un segment de cours d'eau de taille approximative hectométrique. C'est à cette échelle que se font les principales mesures et les échantillonnages qui mènent à la constitution des indices (Indice Biologique Global, Indice Poisson). Une dernière échelle correspond à l'ambiance et permet d'étudier les relations entre le micro-habitat et le poisson.

C'est dans ce contexte d'emboîtement d'échelles géographiques, revisité en hiérarchie des écosystèmes par les biologistes (O’Neill et al., 1986; Ahl et Allen, 1996; Cohen, 1998; Andriamahefa, 1999), que doit être replacée la méthodologie de la pêche électrique. Celle-ci peut être définie comme un comptage des espèces de poissons d'une station, capturés au moyen d'une épuisette grâce à l'inhibition des organismes provoquée par un courant électrique produit dans l'eau par un matériel spécifique et qui agit sur les fibres nerveuses. La précision du protocole peut être de deux ordres. Soit, il s'agit d'un inventaire consistant en un recensement complet de la station. Dans ce cas, la pêche compte tous les poissons sur une surface fluviale dont la longueur correspond au moins à dix fois la largeur du cours d'eau, en s'appuyant sur deux passages successifs, ou plus. Ceux-ci permettent de capturer les poissons qui auraient échappé à la première prise du fait d'une mauvaise position dans le champ électrique ou à cause de leur biologie propre, les fouisseurs étant ainsi rarement pris dès le premier passage. Soit, il s'agit d'un sondage consistant en un comptage partiel censé être représentatif de la population de la station. Celui-ci est effectué sur un espace complet ou bien selon une prospection discontinue reposant sur les habitats potentiels. L'initiation des auteurs à la technique de la pêche électrique a permis d'avoir un œil critique concernant par exemple les approximations, dans le cas du sondage, ou les limites du matériel pouvant conduire à la tétanie ou, au contraire, à l'excitation du poisson. Cela a ensuite servi à déceler, à la source, quelques données aberrantes.

L'essentiel du problème géographique de la méthode réside dans la difficulté à passer d'un échantillonnage ponctuel à une représentativité continue et de la mesure analytique à la cartographie. C'est ici qu'intervient notre premier apport. L'objectif de ce travail a ainsi une double originalité, d’une part, celle de faire entrer la géographie dans un domaine technique, délaissé par les recherches académiques, d'autre part, celle de spatialiser d'une manière synthétique des données dont la cartographie est pour l'instant potentielle et analytique. 
En effet, si, officiellement, la base de données informatisée BDMAP enregistre toutes les pêches effectuées en France par le CSP (puis l'ONEMA) depuis les années 1980, avec indication de la longueur, du poids et de la pathologie externe de chaque poisson ou lot de poissons, dans la pratique notre première étape méthodologique fut la collecte de tous les procès-verbaux des pêches électriques réalisées dans le département de la Creuse sur sept ans : 35 en 1996, 28 en 1997, 37 en 1998, 48 en 1999, 61 en 2000, 66 en 2001, 40 en 2002, soit un total de 315 comptes rendus officiels sous format papier. Cette période, pendant laquelle le protocole est resté le même, a été dépouillée pour nous permettre de déceler une évolution dans le temps. Il faudra cependant se garder de conclusion tranchée, car la durée n'atteint qu'un quart d'une normale hydroclimatique. A ce sujet, nous posons comme postulat, pour tenter d'isoler les autres facteurs explicatifs, le fait que les conditions hydroclimatiques ont été globalement constantes pendant les sept années étudiées. En effet, à la station Météofrance de Guéret-Bongeot (46¹0’48” N, 152’42” E, 402 m), la température moyenne hepta-annuelle a été de $11,1^{\circ} \mathrm{C}$, le léger rafraîchissement des étés sur la période compensant le réchauffement des hivers. Le total des précipitations a été de $1080 \mathrm{~mm}$ par an. En dehors de 1996 (909 mm) et 1999 (1250 mm), les autres années se sont peu écartées de la moyenne. Il n’y a eu ni étiage sévère, ni crue exceptionnelle pendant la période considérée.

Les renseignements ont été ensuite saisis par informatique pour constituer une base de données comprenant non seulement la population piscicole et la description de toutes les stations, mais aussi le protocole de chaque pêche électrique. Cette information est liée à la base géographique Carthage dans un SIG. Ainsi repérées dans l'espace, les données sont traitées, dans un premier temps de façon à extraire une cartographie analytique, espèce par espèce, et ponctuelle, par station du réseau hydrographique. Chaque lieu et chaque date sont renseignés par la présence ou l'absence de l'espèce. La troisième étape consiste à sélectionner les espèces les plus sensibles à un changement des conditions du milieu aquatique, pour lesquelles l'évolution spatiale est manifeste, et à synthétiser ces variations. Deux cartes d'associations sont alors possibles, l'une temporelle, l'autre spatiale. Le premier regroupement est celui de deux ou trois années successives pendant lesquelles les présences ou absences sont les mêmes dans la plupart des stations. Dans l'autre option, il s'agit de réunir les points en isochrones. Pour tracer les isolignes à l'échelle de tout un département, notre interpolation a tenu compte de la différence entre les grands cours d'eau et les ruisseaux affluents. En outre, nous avons augmenté le nombre de données issues de pêches tournantes qui ne repassent pas chaque année au même endroit en posant les deux principes suivants. Dans le cas d'une espèce représentative de l'élargissement de l'espace colonisé en relation avec la dégradation de la qualité des eaux, s’il y a présence l'année n, alors il y a présence l'année $\mathrm{n}+1$ et s'il y a absence l'année $\mathrm{n}$ alors il y a absence l'année $\mathrm{n}-1$. Enfin, nous avons postulé que s'il y a présence dans l'affluent, alors il y a présence dans le cours principal.

Ce croisement interdisciplinaire de méthodes géographiques et biologiques et cette démarche associant une technique appliquée de terrain et une approche académique ont permis d'étudier l'évolution spatiale de la qualité piscicole des cours d'eau de l'ensemble du département de la Creuse.

\section{La régression de la qualité piscicole des cours d'eau vers l'amont}

Le travail cartographique permet de souligner que les tronçons de tête de bassin ne sont souvent plus occupés par l'association originelle de la truite fario, du chabot, du vairon et de la loche franche. Ces espèces lithophiles, qui peuplent les eaux courantes fraîches et bien oxygénées, à substrat grossier, sont un bon indicateur de la qualité de l'habitat. Chaque espèce a cependant ses exigences particulières et, par exemple, la loche franche supporte beaucoup mieux la richesse organique du milieu. En outre, la simple présence n'est pas forcément synonyme de reproduction sur l'ensemble du cycle et les lâchers doivent être pris en compte. Mais l'étude de l'association donne des informations spatiales assez fiables.

A contrario, d'autres espèces de poissons, moins nobles, sont des indicateurs d'une certaine dégradation du milieu aquatique quand leur aire de répartition s'agrandit. Ce sont souvent 
des poissons supportant une oxygénation plus basse, une température plus élevée, parfois une turbidité plus importante. Il n'est pas forcément pertinent de suivre les espèces exotiques, introduites en France, échappées des plans d'eau et si résistantes et ubiquistes qu'elles ne permettent finalement pas de tracer l'évolution spatiale progressive, mais montrent une diffusion généralisée à partir de sources multiples. C'est le cas du poisson-chat. D'autres espèces sont plus intéressantes pour le traçage cartographique, comme le gardon ou le rotengle, car elles peuplent à l'état naturel les cours d'eau les plus importants dans leur partie aval. Mais leur présence dans le chevelu amont est liée aux vidanges ponctuelles de plans d'eau et leur suivi n'a pas une signification progressive. Plus pertinente encore est l'étude des espèces autochtones, qui n'occupent pas naturellement les têtes de bassin, mais qui, sans être des salmonidés, sont tout de même des cyprinidés d'eau vive. Leur éventuelle expansion vers l'amont se fait moins rapidement et concerne réellement une remontée progressive, qui peut être suivie géographiquement et est représentative d'une lente dégradation de la qualité des eaux. Ce dernier choix constitue une originalité de notre recherche par rapport à la prise en compte, plus classique, des seules espèces les plus nobles, qui possèdent des limites d'utilisation en tant qu'indicateur, comme cela a été montré par R. Berthold (2003).

Le chevesne (Leucistus cephalus) est l'un de ces précieux indicateurs. Ce cyprinidé rhéophile indigène, très répandu en aval, ne peuple normalement pas les têtes de bassin. Mais sa remarquable capacité d'adaptation à la modification des habitats lui permet de remonter vers l'amont dès que les conditions physico-chimiques ou morpho-sédimentologiques des cours d'eau se dégradent. La vandoise (Leucistus leucistus) a des qualités assez proches, mais elle permet un traçage moins complet, car, très sensible aux parasites, elle est moins répandue et le faible nombre de pêches électriques où elle est décelée réduit les possibilités cartographiques.

La carte descriptive de l'absence ou de la présence du chevesne lors des pêches électriques pendant sept ans montre qu'au milieu des années 1990 cette espèce ne se retrouve pratiquement pas dans le département de la Creuse (fig. 2). Cela confirme son absence classique dans les eaux fraîches de tête de bassin. En 1996, seuls les grands axes sont remontés, c'est-à-dire la Creuse, le Taurion, la Tardes et son affluent la Voueize. La succession des années montre une diffusion de l'espèce à travers le réseau hydrographique, surtout si l'on effectue un travail cartographique de regroupement des années, sur le critère de l'équilibre entre le nombre de données (fig. 3). Les deux dernières années de l'étude correspondent à une forte hausse de présence du chevesne sur l'ensemble du département. Mais la localisation des pêches, effectuées à des endroits différents selon les années, rend difficile une lecture directe plus approfondie.

La carte interprétative des isochrones de remontée du chevesne met en évidence la colonisation progressive de toute la moitié nord du département (fig. 4). Ce fut entre 1998 et 2000 que la progression spatiale fut la plus importante (fig. 4), alors que la seule étude du pourcentage de présence laissait penser à une évolution plus rapide en 2001-2002 (fig. 3). La Petite Creuse a été un chemin de propagation majeur, l'autre voie de pénétration, depuis l'ouest, ayant été la Gartempe. En revanche, sur la Creuse elle-même, dans son cours supérieur, la limite de présence du chevesne s'est stabilisée.

Les cartes d'autres poissons traceurs de la remontée vers l'amont d'une certaine dégradation de la qualité des eaux, moins démonstratives prises une par une, confirment néanmoins les grandes tendances de celle du chevesne et en nuancent certaines. Par exemple, la vandoise était déjà présente dans la Creuse, la Sédelle et la Voueize au début de l'étude, et elle n’a remonté le Taurion qu'avec un certain retard.

Quant aux cartes des poissons traceurs de la rétractation d'une bonne qualité des eaux, elles sont souvent plus difficiles à interpréter. Mais certaines sont riches d'enseignement. La carte du vairon (Phoxinus phoxinus) montre que ce poisson est absent de nombreuses pêches électriques au cours de ces dernières années dans la Gartempe, la basse Sédelle et, de manière moins nette dans la Petite Creuse, alors que ce n'était pas le cas auparavant (fig. 5). Il faut cependant rester prudent dans l'interprétation, du fait que les populations de vairon, de même, d'ailleurs, que celles de 


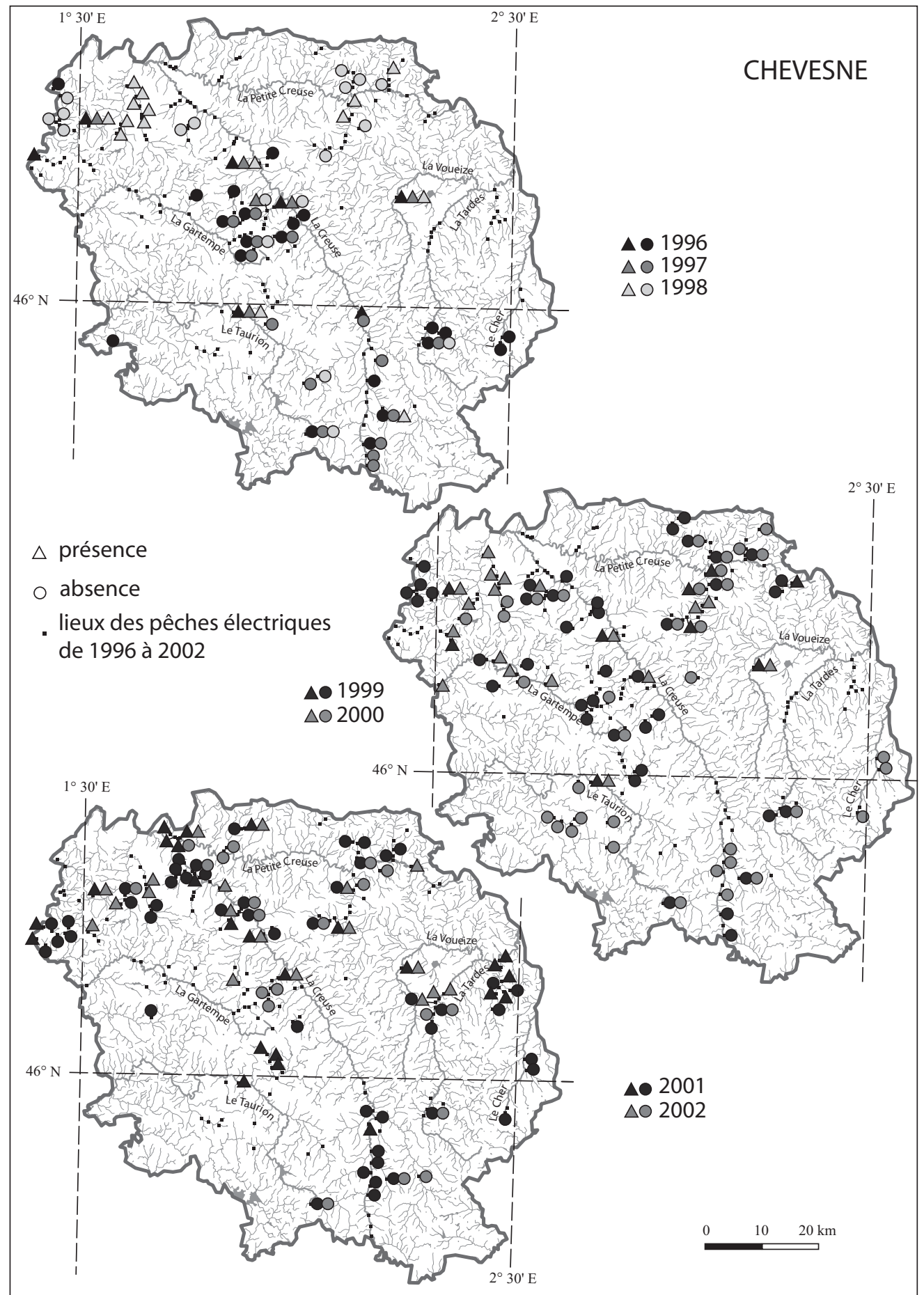

Figure 2 : Carte de l'absence ou de la présence par année du chevesne en Creuse (mesures CSP, conception Clavé Y., Touchart L., réalisation Maillardet J.)

Map of annual absence or presence of chubs in the Creuse department 


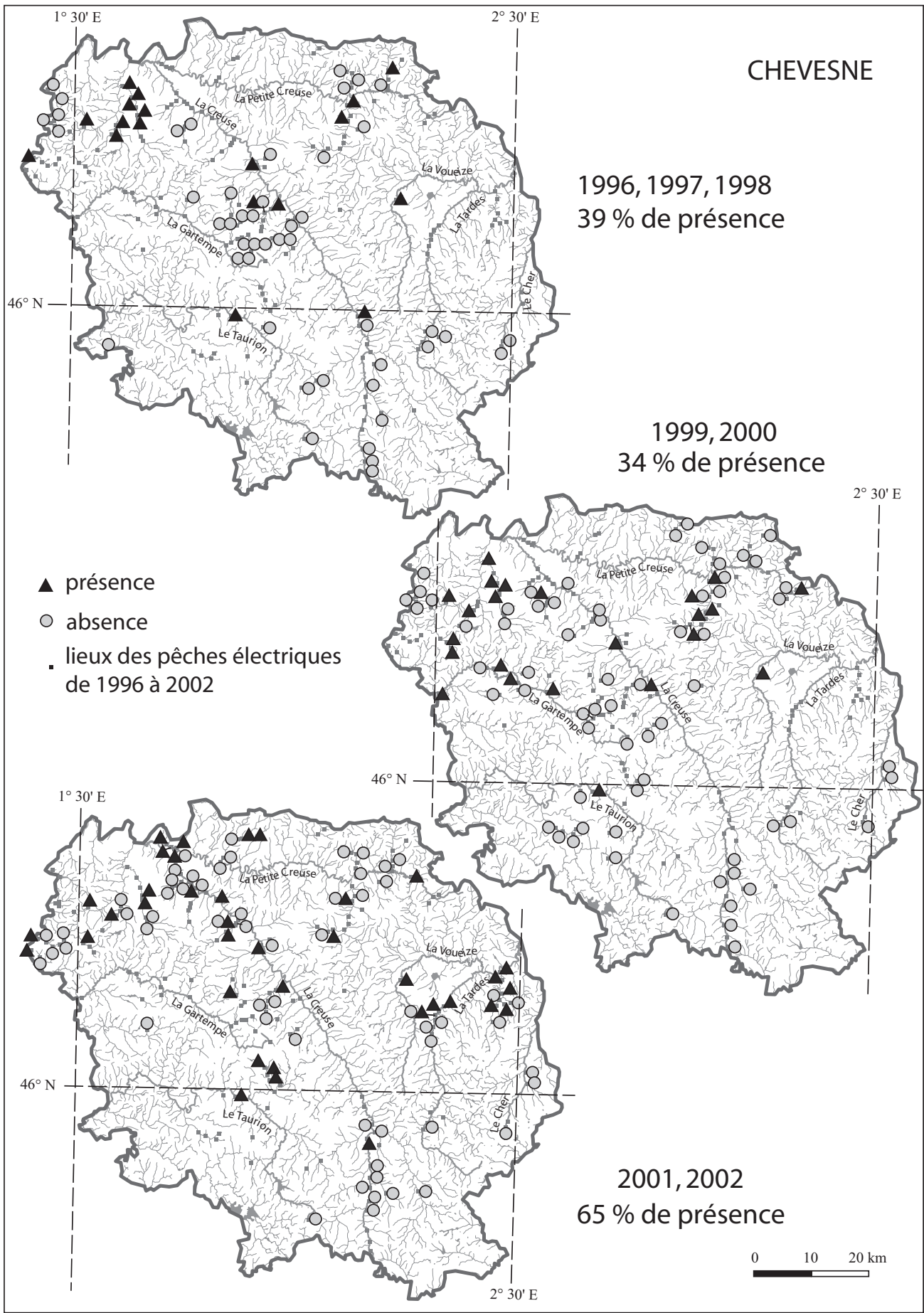

Figure 3 : Carte de l'absence ou de la présence par groupe d'années du chevesne en Creuse (mesures CSP, conception Clavé Y., Touchart L., Maillardet J., réalisation Maillardet J.)

Map of multi-annual absence or presence of chubs in the Creuse department 


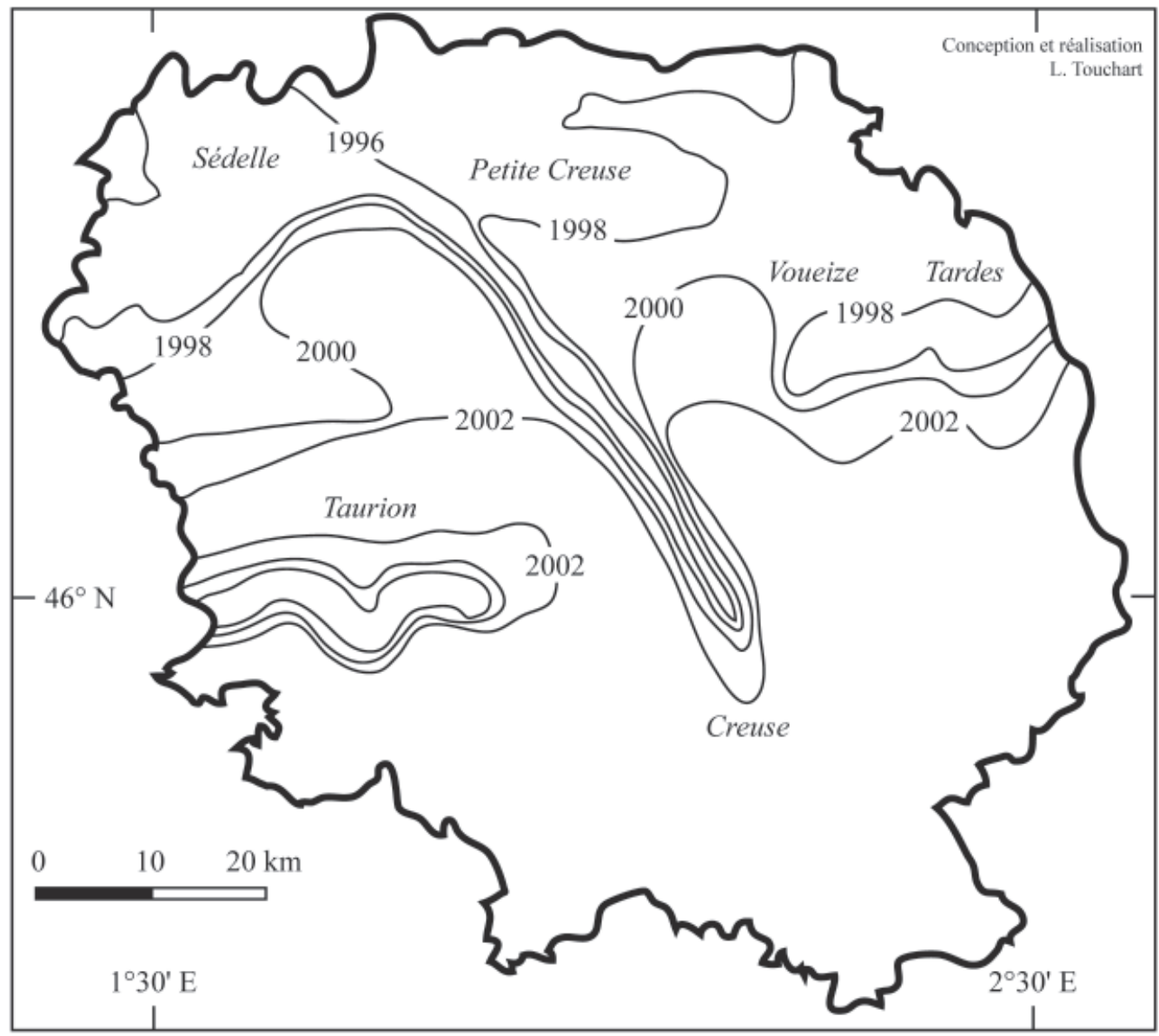

Figure 4 : Carte des isochrones de remontée du chevesne dans le département de la Creuse (conception et réalisation Touchart $\mathrm{L}$.)

Map of isochronal lines showing upstream movement of chubs in the department of the Creuse

goujon, sont plutôt cycliques. Le rôle des crues et des zones de refuge sont fondamentales pour expliquer la présence de ces poissons à longévité assez réduite.

Au total, le référencement et la cartographie analytique de 29 espèces de poissons nous ont permis de tenter une synthèse géographique à l'échelle de l'ensemble du département (fig. 6). Au milieu des années 1990, certains grands cours d'eau possédaient déjà un mélange d'espèces exigeantes et d'espèces vivant dans des conditions d'habitat moins rigoureuses. Il s'agissait de la Creuse en aval d'Ahun, de la Sédelle, du Taurion et de la Voueize. Lors de la dernière décennie, les espèces peu exigeantes ont colonisé le nord et le centre-ouest du département, en remontant les bassins de la Gartempe et de la Petite Creuse. Cela corrobore le diagnostic du PDPG (Fédération de la Creuse, 2008), mais y ajoute le facteur du temps. Dans le cas de la Gartempe, notre recherche montre que la perturbation des contextes intermédiaires de l'aval et salmonicoles de l'amont est récente. Pour simplifier, cette situation concerne maintenant les deux tiers nord-ouest du département et relègue le peuplement exclusif des espèces les plus exigeantes au tiers sud-est. Dans le détail cependant, certains bassins importants du nord-ouest restent préservés, comme celui de l'Ardour, entre Gartempe et Taurion. Cela pose donc la question des causes de cette évolution. 


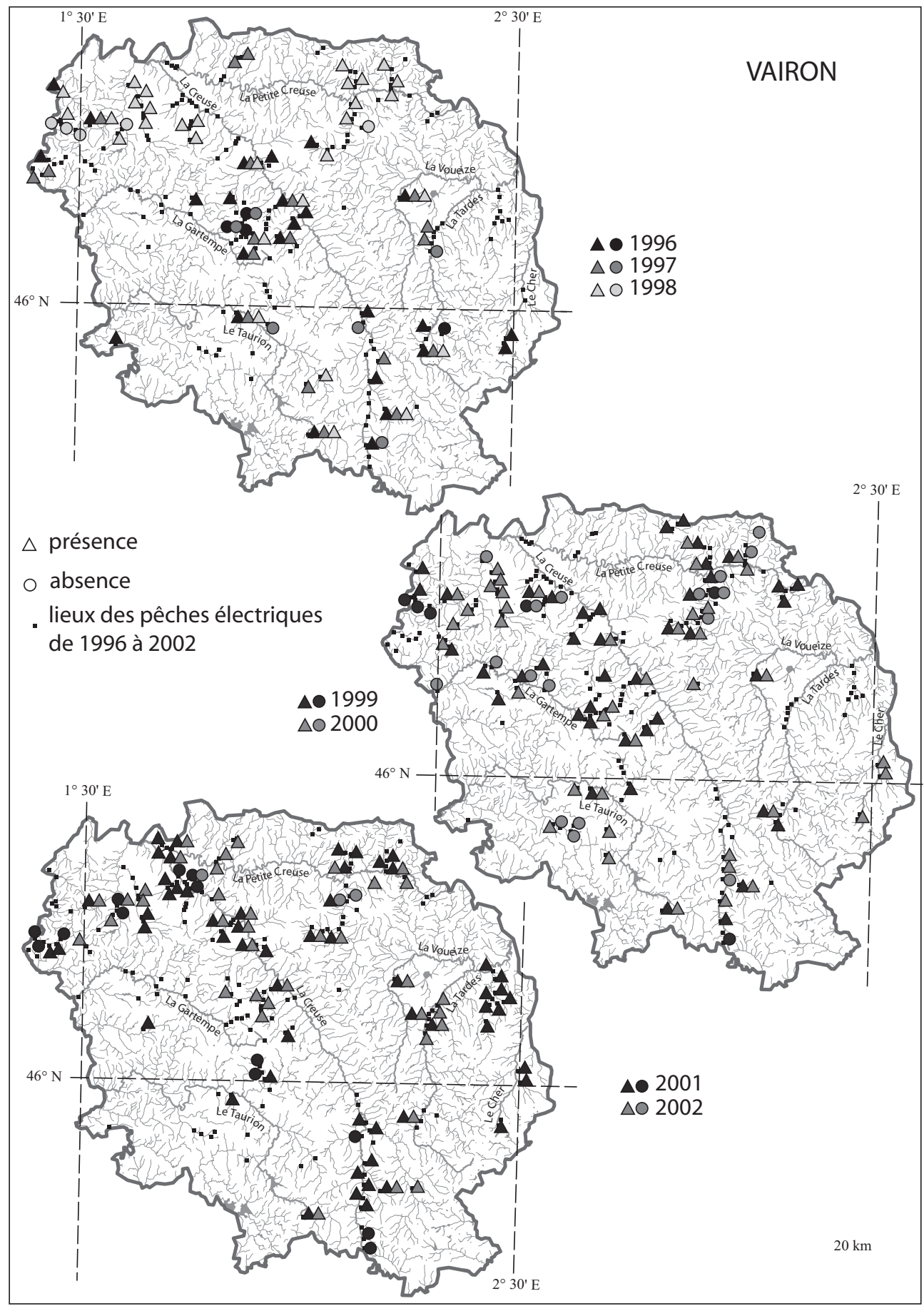

Figure 5 : Carte de l'absence ou de la présence par année du vairon en Creuse (mesures CSP, conception Clavé Y., Touchart L., réalisation Maillardet J.)

Map of annual absence or presence of minnows in the Creuse department 


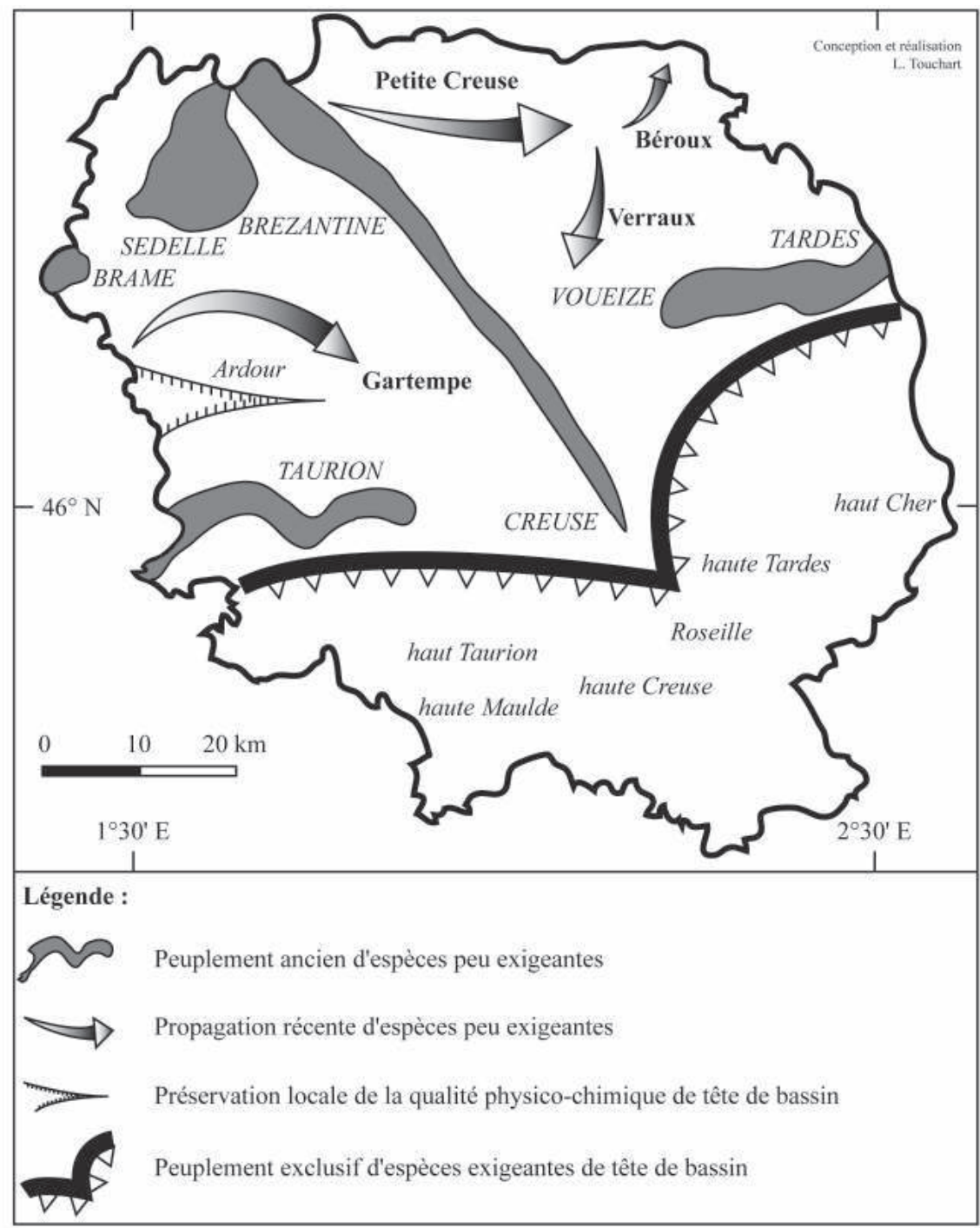

Figure 6: Carte de l'évolution piscicole des cours d'eau creusois, une géographie de tête de bassin de moyenne montagne (conception et réalisation Touchart L.)

Map of piscicultural changes in watercourses of the Creuse department, a geography of mid-alpine headstream waters

Les vidanges de plans d'eau et la multiplication du nombre d'étangs pourraient expliquer certaines modifications du peuplement piscicole des cours d'eau du département. En effet, les régions où la croissance des plans d'eau a été la plus forte lors des dernières années, notamment le nordest du département et la partie amont du bassin de la Petite Creuse, correspondent à celles d'une diffusion progressive des espèces les moins exigeantes, au moins en terme de température de l'eau. Il existe maintenant 5579 plans d'eau dans le département, dont 3804 étangs de plus de 10 ares, et, parmi eux, 2764 sont en lien direct avec le chevelu hydrographique (Bartout, 2006). Au contraire, les pêches sont restées les plus proches de leurs résultats initiaux dans le bassin de l'Ardour dans lequel on observe une stagnation du nombre d'étangs. Mais des changements plus 
amples, affectant l'ensemble du département, offre également de nouvelles conditions de remontée progressive et généralisée des espèces les moins exigeantes : une population rurale déclinante, un entretien moins régulier des berges des cours d'eau favorisant les accumulations sédimentaires, un enrésinement croissant vers l'amont et un léger réchauffement des hivers sur la période étudiée.

\section{Conclusion}

Appuyée sur le croisement de méthodologies fondamentale et appliquée et l'apport interdisciplinaire de démarches géographique et biologique, cette recherche associe le travail des techniciens de terrain et celui de la cartographie universitaire. À l'échelle de l'ensemble d'un département couvrant $5600 \mathrm{~km}^{2}$, elle permet de spatialiser un type de données trop peu usité en géographie, celui des résultats des pêches électriques, puis d'interpréter ces cartes en terme d'évolution de la qualité des eaux et, plus largement, des milieux. Cette recherche contribue à dépasser les indicateurs classiques à la disposition des géographes afin de proposer une réflexion concernant le développement durable et œuvrer activement pour construire une gestion raisonnée de l'environnement. Le nouveau Parc Naturel Régional de Millevaches, qui couvre le sud du département de la Creuse, gère un territoire dont nos travaux montrent la bonne santé du chevelu hydrographique et la présence d'une population piscicole exigeante. Ce type de régions, celles des têtes de bassin de moyenne montagne comme le Massif central et les Vosges, peut devenir le refuge des espèces prisées et acquiert ainsi une nouvelle valeur et une certaine responsabilité.

\section{Bibliographie}

Ahl (V.), Allen (T. F. H.), 1996. - Hierarchy theory: a vision, vocabulary and epistemology, Columbia University Press, 206 p.

Amoros (C.), Petts (G. E.) (dir.), 1993. - Hydrosystèmes fluviaux, Paris, Masson, 300 p.

Andriamahefa (H.), 1999. - Les hydro-écorégions du bassin de la Loire. Morphologie, hydrologie, pressions anthropiques sur les cours d'eau et les bassins-versants, thèse de doctorat, Université Jean-Monnet, SaintÉtienne, $272 \mathrm{p}$.

Ardillier-Carras (F.), 1997. - L'eau ressource pour le développement d'un espace rural, l'exemple du bassin de la Gartempe, Limoges, Pulim, 592 p.

Balabanian (O.), Bouet (G.), 1989. - L'eau et la maîtrise de l'eau en Limousin, Treignac, Les Monédières, $302 \mathrm{p}$.

Bartout (P.), 2006. - Pour un référentiel des zones humides intérieures de milieu tempéré : l'exemple des étangs en Limousin (France), typologies, régionalisation, thèse de doctorat en géographie, Université de Limoges, 497 p.

Berthold (R.), 2003. - Méthodologie pour la gestion durable des têtes de bassins versants, de l'analyse globale des bassins versants au diagnostic écologique des systèmes ruisseaux, Besançon, $6^{\mathrm{e}}$ Rencontres de Théo Quant [http://thema.univ-fcomte.fr/theoq/pdf/2003/03Berthold.pdf], 11 p.

Brun (A.), Pinet (J.-M.), 2004. - « Gestion du cheptel piscicole et surveillance des milieux aquatiques », Le Courrier de l'environnement de l'INRA, n 51, p. 31-42.

Bravard (J.-P.), 1987. - Le Rhône du Léman à Lyon, Lyon, La Manufacture, 451 p.

Carré (F.), 1978. - «Les pêches en mer Caspienne », Annales de Géographie, vol. 87, n 479, p. 1-39.

Cohen (P.), 1998. - Régionalisation de l'habitat physique du poisson. Approche multi-scalaire et application au bassin de la Loire, France, thèse de doctorat, Université Claude-Bernard, Lyon, 266 p.

Descamps (H.), Naiman (R. J.), 1989. - «L'écologie des fleuves », La Recherche, vol. 20, n² 208, p. 310-319.

Fédération de la Creuse pour la Pêche et la Protection du Milieu Aquatique, 2008. - Plan Départemental pour la Protection du milieu aquatique et la Gestion des ressources piscicoles de la Creuse, Guéret, 339 p. 
Fédération des PNR de France, pôle-relais zones humides intérieures, 2007. - Actes des rencontres nationales techniques. Gestion des ruisseaux de tête de bassin et zones humides associées, Saint-Brisson, Maison du Parc Naturel Régional du Morvan, 179 p.

Girard (P.), 1998. - « Le poisson sentinelle de milieux aquatiques : pertinence et optimisation des indicateurs sanitaires », Bulletin français de la Pêche et de la Pisciculture, vol. 71, n 350-351, p. 429-443.

Huet (M.), 1949. - «Aperçu des relations entre la pente et les populations piscicoles des eaux courantes », Schweizerische Zeitschrift der Hydrologie, vol. 11, n 3-4, p. 332-351.

ILLIES (J.), 1955. - «Der biologische Aspekt der limnologischen Fliessgewässer », Archiv für Hydrobiologie, Suppl., vol. 22, p. 337-346.

—, 1961. - «Versuch einer allgemeinen biozönotischen Gliederung der Fliessgewässer », Internationale Revue der gesamten Hydrobiologie, vol. 46, p. 205-213.

Illies (J.), Botosaneanu (L.), 1963. -, « Problèmes et méthodes de la classification et de la zonation écologique des eaux courantes considérées surtout du point de vue faunistique », Mitteilungen der Internationale Vereinigung für theorische and angewandte Limnolologie, vol. 12, p. 1-57.

Karr (J. R.), 1981. - «Assessment of biotic integrity using fish communities », Fisheries, n ${ }^{\circ}$ 6, p. 21-27.

Keith (P.), Allardi (J.), 2001. - Atlas des poissons d'eau douce de France, Paris, Muséum National d'Histoire Naturelle, coll. « Patrimoines naturels », n 47, 387 p.

Oberdorff (T.), Pont (D.), Hugeny (B.), Belliard (J.), Berrebit dit Thomas (R.), Porcher (J.-P.), 2002a. - «Adaptation et validation d'un indice poisson (FBI) pour l'évaluation de la qualité biologique des cours d'eau français », Bulletin Français de Pêche et Pisciculture, n 365/366, p. 405-433.

Oberdorff (T.), Pont (D.), Hugeny (B.), Porcher (J.-P.), 2002b. - « Development and validation of a fish-based index (FBI) for the assessment of "river health" in France », Freshwater Biology, vol. 47, n 9, p. 1720-1734.

O’Neill (R. V.), Deangelis (D. L.), Waide (J. B.), Allen (T. F. H.), 1986. - A hierarchical concept of ecosystems, Princeton University Press, Monographs in Population Biology n² 23, 262 p.

Persat (H.), Keith (P.), 1997. - « The geographic distribution of freshwater fishes in France: which are native and which are not? ", Bulletin Français de Pêche et Pisciculture, n ${ }^{\circ}$ 344/345, p. 15-32.

Rougerie (G.), 1993. - Biogéographie des milieux aquatiques, Paris, A. Colin, 252 p.

Schéma d’Aménagement et de gestion des eaux du bassin versant Cher amont, 2007. - Etat des lieux de la ressource en eau, des milieux et des usages, Montluçon, Geo-Hyd, 238 p.

Vannote (R. L.), Minshall (G. W.), Cummins (K. W.), Sedell (J. R.), Cushing (C. E.), 1980. - « The river continuum concept », Canadian Journal of Fisheries and Aquatic Science, vol. 37, p. 130-137.

Verneaux (J.), 1977. - «Biotypologie de l'écosystème 'eau courante'. Détermination approchée de l'appartenance typologique d'un peuplement ichtyologique », Comptes rendus de l'Académie des Sciences, vol. 284, p. 675-678.

Wasson (J.-G.), Bethemont (J.), Degorce (J.-N.), Dupuis (B.), Joliveau (T.), 1993. - Approche écosystémique du bassin de la Loire : éléments pour l'élaboration des orientations fondamentales de gestion. Phase I : état initial - problématique, Lyon et Saint-Étienne, Cemagref et Université Jean Monnet, 102 p. + 70 planches.

\section{Remerciements}

Les auteurs remercient Monsieur L. Dubois, garde-chef de la brigade du CSP de la Creuse, pour la fourniture de tous les procès-verbaux et l'initiation aux pêches de terrain, Monsieur P. Bartout, docteur en géographie, pour la communication de données inédites concernant le recensement des étangs, Monsieur F. Carré, Professeur de géographie de la mer à l'Université de Paris IV Sorbonne, pour ses informations bibliographiques concernant la géographie de la pêche, et Monsieur D. Monnier, membre du pôle-relais «zones humides intérieures » et de la Délégation Régionale de Metz du Conseil Supérieur de la Pêche (Onema), pour la fourniture de la bibliographie la plus récente, sa relecture attentive du manuscrit et ses judicieuses propositions d'amélioration. 